\title{
Time reversed acoustics techniques for elastic imaging in reverberant and nonreverberant media: An experimental study of the chaotic cavity transducer concept
}

\author{
Bart Van Damme, ${ }^{1, a)}$ Koen Van Den Abeele, ${ }^{1}{ }^{\text {YiFeng }} \mathrm{Li},{ }^{2}$ and Olivier Bou Matar ${ }^{3}$ \\ ${ }^{1}$ Wave Propagation and Signal Processing Research Group, K.U.Leuven Campus Kortrijk, E. Sabbelaan 53, \\ B-8500 Kortrijk, Belgium \\ ${ }^{2}$ College of Electronics and Information Engineering, NanJing University of Technology, NanJing, 210009, \\ China \\ ${ }^{3}$ International Associated Laboratory, LEMAC, IEMN, UMR, CNRS, 8520 PRES Lille Nord de France, \\ ECLille, Fr-59652 Villeneuve d'Ascq, France
}

(Received 4 February 2011; accepted 11 April 2011; published online 31 May 2011)

\begin{abstract}
In view of emerging imaging technologies based on the combination of Time Reversed Acoustics (TRA) with Nonlinear Elastic Wave Spectroscopy (NEWS) for the detection and localization of micro-damage in solids, we have investigated the benefits of chirped source signal excitation, inverse filtering techniques, and the implementation of chaotic cavity transducers to improve the quality of energy focusing, especially for weakly reverberant media. Chaotic cavity transducer focusing is defined as the hardware-software combination of a piezoelectric ceramic glued on a cavity of chaotic shape on the one hand with the reciprocal Time Reversal (or Inverse Filter) technique on the other hand. Experimental data for reverberant and nonreverberant composite plates show that the use of chirps, inverse filtering and chaotic cavity transducers significantly enhances the focusing process, and enables focusing in a nonreverberant medium using only one transducer. As a potential exploitation, the application of the chaotic cavity transducer concept for synthetic imaging is examined, revealing several properties similar to phased arrays. (C) 2011 American Institute of Physics. [doi:10.1063/1.3590163]
\end{abstract}

\section{INTRODUCTION}

Time Reversed Acoustics (TRA) techniques provide the ability to focus ultrasonic waves in time and space, regardless of the position of the initial source and the heterogeneity of the medium in which the wave propagates. This technique has attracted great scientific and technological interests in different fields including medical therapy, diagnostic, nondestructive testing (NDT), and underwater acoustics. ${ }^{1}$ In a standard TRA experiment, waves generated by an acoustic source are first measured by an array of reversible piezoelectric transducers located around the source, and then time reversed and re-emitted by the same transducers array. To improve the quality of the focusing the transducers should cover a closed surface around the medium in order to obtain the wave front information coming from all directions. ${ }^{2}$ However, as this is difficult to realize in practice, the time reversal operation is usually performed on a limited angular area, thus adversely affecting the reversal and focusing quality.

The quality of energy focusing in solid media using the principles of TRA is highly influenced by the nature and dimensions of the medium, the number of transducers and the length of the received signals. It has been observed that multiple scattering ${ }^{3}$ and multiple reflections, as in the case of a waveguide ${ }^{4-6}$ or a cavity, ${ }^{7-9}$ tend to enhance the focusing quality both in resolution and in amplitude. As a consequence, in the case of highly reverberant media, the number

\footnotetext{
a) Author to whom correspondence should be addressed. Electronic mail: bart.vandamme@kuleuven-kortrijk.be.
}

of channels participating in the time reversal process can be reduced, even to only one channel as demonstrated by Draeger et al. ${ }^{7-9}$ and Fink et al. ${ }^{10}$ in a silicon wafer chaotic cavity. This astonishing behavior has been linked to the ergodic property of the chaotic cavity, bearing the possibility to collect all information in only one point.

Since classical TRA procedures require transducers that can be used both as emitter and as receiver, a state-of-the-art switching device is needed in order to properly arrange the flow of signals from the generator cards to the sources and from the receivers to the data acquisition cards. To avoid this supplemental cost, reciprocal time reversal techniques are being used. In this case, a signal measured at a particular receiver is first time reversed, and subsequently broadcasted back into the medium through the original emitter. Due to reciprocity, the energy will then focus on the receiver being considered. In the case of more than one receiver, all time reversed signals can be added when the medium is assumed to be linear, resulting in a focusing of the energy on the location of all individual receivers. ${ }^{11,12}$

Over the last decade, innovative ultrasonic methods have been developed to probe the existence of damage (e.g., delaminations, micro-cracks or weak adhesive bonds) by investigating various nonlinear signatures such as the generation of harmonics, the inter-modulation of frequency components, the amplitude dependent shift in resonance frequencies, pulse inversion asymmetry and slow dynamic conditioning. ${ }^{13,14}$ Such approaches are termed Nonlinear Elastic Wave Spectroscopy (NEWS) techniques. The basis of all NEWS techniques is to measure and analyze macroscopic nonlinear 
signatures resulting from a local violation of the linear stressstrain relation at the microscale. ${ }^{14}$ Generally, the passage of a high amplitude wave suffices to trigger the nonlinear response. Tests performed on a wide variety of materials subjected to different micro-damage mechanisms of mechanical, chemical and thermal origin, have shown that the sensitivity of such nonlinear methods to the detection of the fatigue state of a structure is far greater than what can be obtained with linear acoustical methods. The methods have recently been extended to visualization techniques for imaging defect nonlinearity distributions using laser vibrometry, ${ }^{15}$ airborne ultrasound ${ }^{16}$ or shearography. ${ }^{17}$

The fact that TRA can locally induce high amplitudes with high resolution has attracted the interest of the nonlinear acoustics community, and several basic concepts of merging the benefits of both TRA and NEWS have been proposed in order to identify and visualize defects in solid samples. ${ }^{18-20}$ In most NDT applications of this combination, a one channel TRA experiment is performed in which a piezoelectric (PZT) ceramic is glued directly to a sample, and for which the sample itself is generally small enough to be considered as multi-reverberant in the frequency range of interest. ${ }^{18,19}$ Obviously, certain problems may be expected for reverberating systems with a high degree of symmetry, since simple reverberating properties may lead to the concentration of virtual sources on a pattern with dimensions correlated to size of the sample (or the transducer) resulting in spatial diffraction figures and "phantom" images. ${ }^{8,21}$ Furthermore, the application of traditional as well as reciprocal single channel TRA to focus energy in a nonreverberant samples encounters serious difficulties and deficiencies due to a lack of information of reflections.

In this article, we are proposing modifications of the TRA techniques that overcome the above mentioned shortcomings. The key issue is the definition and implementation of a chaotic cavity transducer (CCT) (Sec. II). After describing the CCT concept, two additional modifications related to excitation and signal treatment to improve TRA focusing are proposed and illustrated (Sec. III). The benefits are discussed by means of two sets of experiments. The first set of experiments deals with a reverberating steel plate and shows the strength of the CCT device in eliminating phantom images (Sec. IV A). The second set concerns the application of single channel CCT-TRA to focus energy in a highly damped nonreverberating composite plate (Sec. IV B). Finally, the CCT concept is applied in the same philosophy as a phased array transducer, enabling energy focusing at an arbitrary point in a medium (Sec. IV C).

\section{CHAOTIC CAVITY TRANSDUCER}

In order to overcome the "phantom" image problem and to extend the TRA method to nonreverberating samples, we propose to broaden the concept of "chaotic cavity transducer", originally introduced for $3 \mathrm{D}$ imaging in fluids, ${ }^{22}$ toward applications dealing with elastic waves in solids. The CCT concept simply consists of a combination of a PZT ceramic glued to a cavity of chaotic shape on the hardware side with the time reversal principle on the software side.
Montaldo et l. $^{22}$ showed that a transducer glued on a chaotic cavity can be interpreted as an array of transducers, with potential exploitation for imaging using pressure waves in fluids. Here, we develop the counterpart for focusing elastic waves in solids.

In the experimental implementation for solids, a single PZT ceramic disk is glued to an aluminum chaotic cavity, and, similarly, the cavity is glued directly onto the solid sample. An electrical source signal, generated by an arbitrary waveform generator and amplified by a power amplifier, is applied to the PZT ceramic which generates a mechanical wave that propagates inside the cavity and radiates energy into the sample medium. Each time the wave propagating in the cavity arrives at the boundary between the cavity and sample, part of the incident energy is reflected and continues to engender multiple reflections on the other boundaries of the cavity, whereas the other part of the energy is transmitted in the sample as shown on Fig. 1. A laser vibrometer is used to record the response at an arbitrary point on the surface of the sample, either following the direct emission or after the time reversed excitation. The signals are generated and acquired though a PXI system from National Instruments, and the full reciprocal time reversal process as well as the scanning and positioning of the laser are computer controlled by LabVIEW.

\section{IMPROVING CONTRAST AND QUALITY OF FOCUSING}

In classical TRA, short pulses or burst excitation at a single frequency and regular time reversal rebroadcasting $S_{\text {TRA }}(t)=R_{\text {Direct }}(-t)$ is used. In order to increase both the quality of the focus (amplitude) and the signal to noise ratio (contrast), chirped excitation and time reversal using inverse filtering can be suggested as an improvement over the classical time reversal process.

\section{A. Chirped excitation}

Due to the low amount of total energy in a short pulse, the use of a simple sinusoidal burst for impulse response measurements does not generally provide a strong received signal, leading to a poor signal to noise ratio. To obtain a more robust measurement of the impulse response together with a substantial improvement of the quality of focusing, a more efficient pulse compression technique based on a linear sweep signal should be used instead of the short sinusoidal pulse. As a first step in this procedure, a direct emission is performed using a chirp with linear increasing instantaneous frequency:

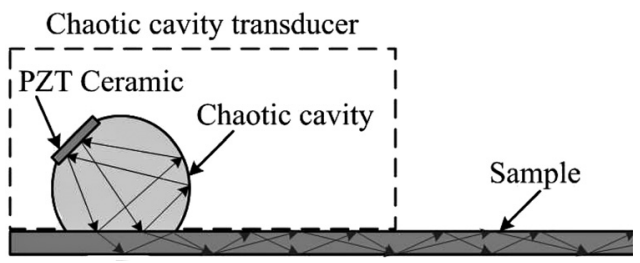

FIG. 1. Principle of the chaotic cavity transducer. 


$$
s(t)=A(t) \cos \left(2 \pi f_{0} t+\pi \frac{B}{T} t^{2}\right),-\frac{T}{2} \leq t \leq \frac{T}{2},
$$

where $f_{0}$ is the center frequency, $T$ is the signal duration, and $B$ is the total bandwidth that is swept. The instantaneous frequency is

$$
f(t)=f_{0}+\frac{B}{T} t
$$

which is a function of time that indicates the spectral band in which the signal energy is concentrated at the time instant $t$. It is known that this kind of chirp is robust to a down-shift in central frequency due to attenuation. ${ }^{23}$ After measuring the waveform at the receiver position, the pulse compression is finally accomplished by taking the cross correlation of the recorded waveform with the time reversed input signal and by rebroadcasting it back into the medium. In order to reduce time domain sidelobes which may appear in the pulse compression process, a time domain Blackman window apodization $A(t)$ can be used.

Figure 2, as an example of a representative result, compares the direct wave recorded signal and the time recompressed signal at the focus after rebroadcasting the convoluted and time reversed signal for two kinds of source signals: a sinusoidal pulse and a linear sweep. In this experiment the typical values used for the linear sweep were $T=100 \mu s, f_{0}=600 \mathrm{kHz}$, and $B=800 \mathrm{kHz}$, and the sinusoidal pulse was a 2 period long sinusoidal burst at frequency $600 \mathrm{kHz}$. It is clear that the use of linear sweep source signal improves the time recompression quality and the signal to noise ratio easily by almost $50-100 \%$.

The use of pulse compression in TRA allows to transfer high levels of energy into the test sample without increasing the peak intensity of the excitation. This is particularly interesting for NEWS based imaging of defects because of the fact that the nonlinear response of a crack generally displays a threshold behavior, and a quadratic increase with amplitude as soon as this threshold is surpassed. ${ }^{15,24}$
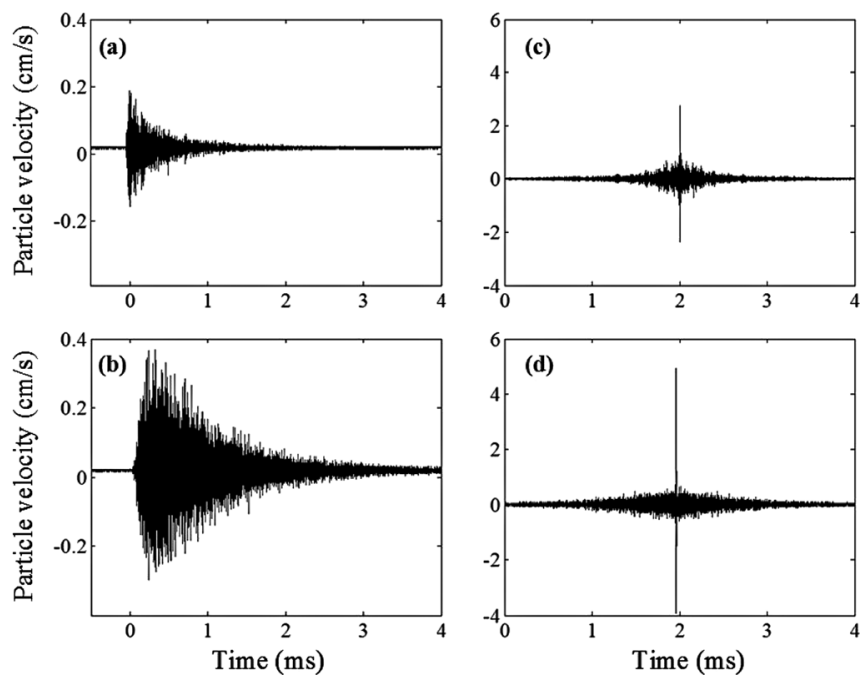

FIG. 2. Comparison of the TR result for a 2-cycle burst source signal (a-c) and a chirped source signal (c-d). Both the direct wave response signal (a-b) and the time reversed signal after pulse compression (c-d) are shown.

\section{B. Inverse filter method}

The spatio-temporal Inverse Filter (IF) method has been introduced by Tanter et al. ${ }^{25}$ and Aubry et al., ${ }^{26}$ and has shown to considerably improve the focusing quality compared to the classical TR method. At the same time, the IF method improves the contrast of the retro-focused signal, which is defined as the ratio between the energy of the signal at the recompression time and the average energy of the signal at all other times. ${ }^{11}$ Typical comparative results of regular TR and IF-TRA are shown in Fig. 3. In this example, the "focus-to-noise" contrast for the IF method has improved by a factor of about two compared to the results of the regular TR method.

A physical interpretation of the contrast in terms of information grains has been given by Derode et al. ${ }^{27}$ An impulse response can be viewed as a recurrence of decorrelated information grains of which the duration is equal to the duration of the initial pulse. The peak of recompression results from the coherent sum of the information grains whereas the surrounding noise is the result of their incoherent sum. The fundamental difference between regular TR and IF-TRA is that the IF approach constructs the signal to be rebroadcasted as a linear combination of all eigenmodes of the sample object, weighted by an inversion of the energy of the respective eigenmode that is present in the received direct signal. ${ }^{11}$ Doing so, the IF focusing process takes advantage of all the modes including those with the weakest energy which are poorly exploited in the regular classical time reversal focusing process. The weighted eigenmode spectra for both approaches corresponding to the result shown in Fig. 3 are illustrated in Fig. 4. The IF approach uses a more homogeneous spectrum than regular TR.
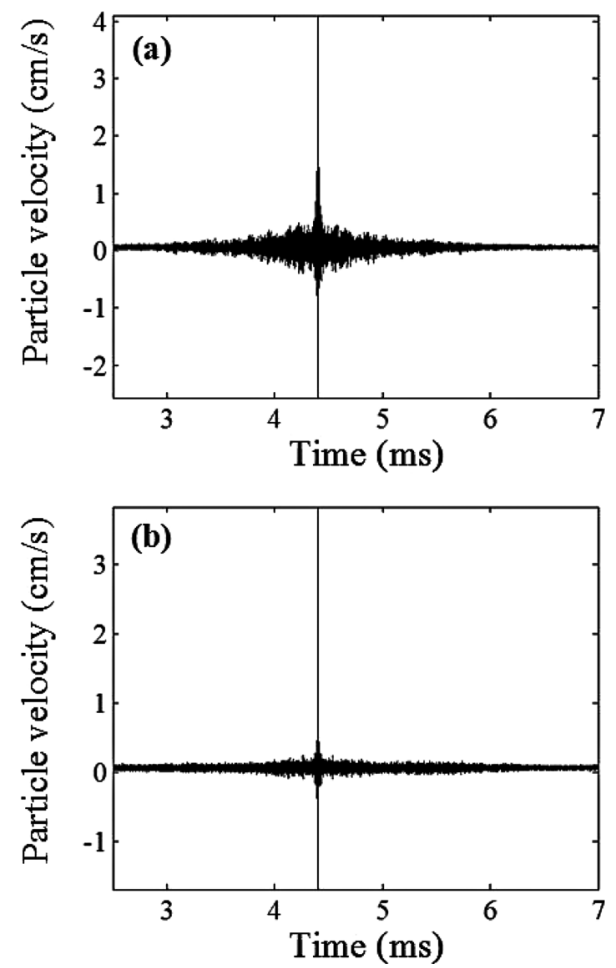

FIG. 3. Comparison of the TR result without (a) and with (b) inverse filtering. 

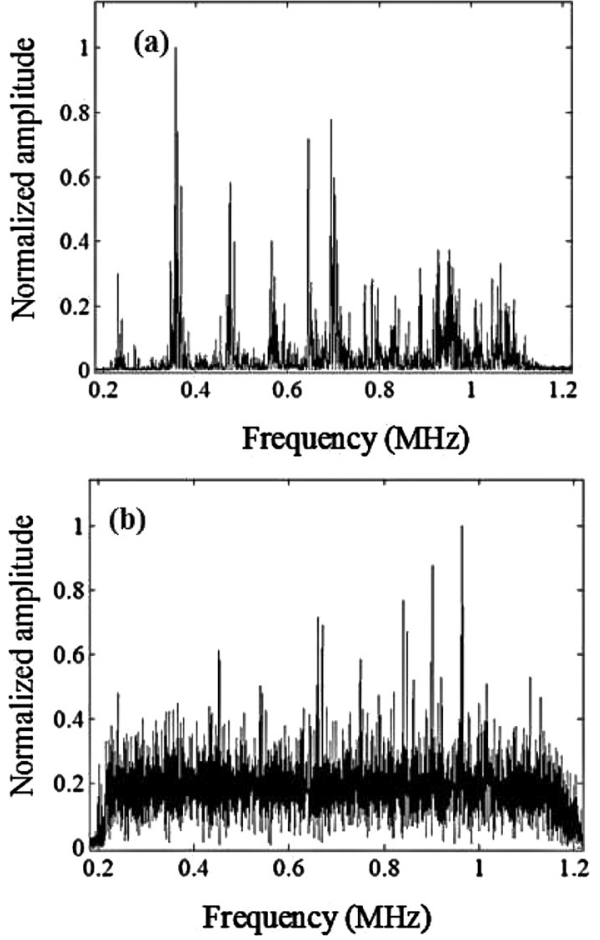

FIG. 4. Comparison of the spectra of the time-recompression signal obtained with regular Time Reversal (a) and Inverse Filtering (b) methods.

As an example, Fig. 5 shows experimental data of the "focus-to-noise" contrast, measured in the case of a copper chaotic cavity glued on a $12 \times 2.5 \times 1.0 \mathrm{~cm}^{3}$ rectangular steel sample, as a function of the duration $\Delta T$ of the time reversed or inverse filtered signal. The overall evolution of the observed contrast starts with a linear increase with signal duration, followed by a marked saturation, and is in complete accordance with the theoretical description reported by Quieffin. ${ }^{11}$

\section{EXPERIMENTS ON PLATE STRUCTURES, RESULTS AND DISCUSSION}

\section{A. Reducing phantom images}

In systems with a high degree of symmetry, the recombination of highly symmetrical eigenmodes after a simple TR process may result in the appearance of phantom images and boundary effects. One of the main advantages of using

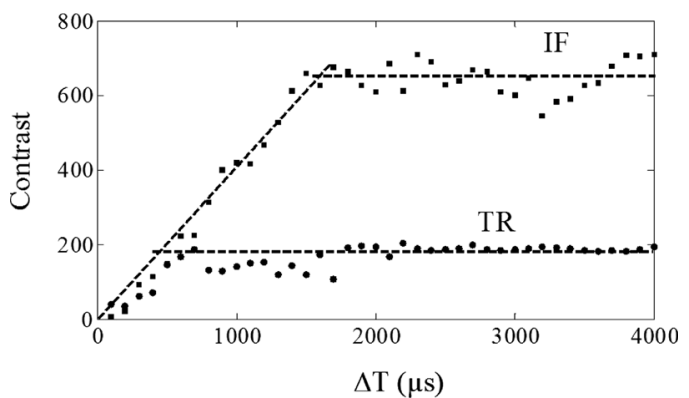

FIG. 5. Contrast of the retro-focused signal for Inverse Filter (IF) and regular Time Reversal (TR) processes as a function of the source signal length, showing an improvement by a factor of three for the inverse filtering technique. chaotic cavities is that the manifestation of virtual sources and confusing hot-spots can be eliminated. Indeed, by definition, the chaotic nature of the cavity will break any obvious or hidden symmetry of the problem, and this will automatically result in an unambiguous retro-focusing. To illustrate this we performed a CCT-TR experiment on a $12 \times 2.5 \times 1.0 \mathrm{~cm}^{3}$ rectangular steel sample, on which we have glued a copper chaotic cavity. A PZT ceramic bonded on the cavity is excited by a chirped signal. The corresponding reverberating signal is recorded at an arbitrary position on the surface of the sample using a laser vibrometer (only the out-of plane component is measured). We then perform a reciprocal IF signal process to focus the elastic wave energy at the considered point on the surface of the steel sample.

The results for the case where the intended focal position is $7.5 \mathrm{~mm}$ away from the boundary are shown in Figs. 6 and 7. Snapshots of a 2D scan at 5 time instances (before, on and after focus-time) are displayed for a $15 \times 15 \mathrm{~mm}^{2}$ surface area around the intended focal point in Fig. 6, demonstrating the high quality of the retro-focusing, with a spot size of $2 \mathrm{~mm}$ radius. The spatial distribution of the focused signal in two orthogonal directions [Fig. 7(a)], perpendicular and parallel to the boundary, clearly shows that there is no increase of the strain at the stress free boundary (which is the upper boundary on Fig. 6). The resulting focal spot is much cleaner and the contrast is enormously improved compared to the case when a PZT ceramic is directly glued on the reverberating sample without intermediate chaotic cavity. ${ }^{19,20}$ Similar excellent focusing results can be obtained when the intended focal point is exactly on a boundary of the sample [Fig. 7(b)], demonstrating the ability of the CCT to focus even on the border of the material sample.

\section{B. Time reversal focusing in nonreverberant media}

The combination of reciprocal single channel TRA with nonreverberant samples poses serious problems for the technique as the sample itself cannot be used as a reflecting body thereby creating the necessary virtual sources. In this case, the utilization of a CCT offers a suitable solution to extend the possibility of focusing energy in such sample without having to invest into a multichannel TRA system. To illustrate this, a similar reciprocal reversal experiment as discussed before for the multi-reverberating steel sample was performed on a nonreverberant composite plate with relatively large dimensions $\left(65 \times 65 \times 0.3 \mathrm{~cm}^{3}\right)$. A CCT $(\mathrm{PZT}$ disk glued to an aluminum cavity) was bonded on the composite plate near its center. The contact area cavity-plate is about $6 \times 1 \mathrm{~cm}^{2}$. The PZT was excited with a $100 \mu$ s long sweep for which the center frequency $f_{0}=100 \mathrm{kHz}$, and the bandwidth $B=200 \mathrm{kHz}$. The out-of plane signal at a fixed point on the surface of the composite plate away from the cavity is measured by a laser vibrometer and sent back into the medium by the same PZT after the IF-TR pulse compression convolution process.

A 2D scan of the amplitude of the resulting wave field in the region of the chosen fixed point, on a square area of $50 \times 50 \mathrm{~mm}^{2}$ with a spatial step of $1 \mathrm{~mm}$, is plotted in Fig. 8(b), for three time instances: before, at, and after the exact 

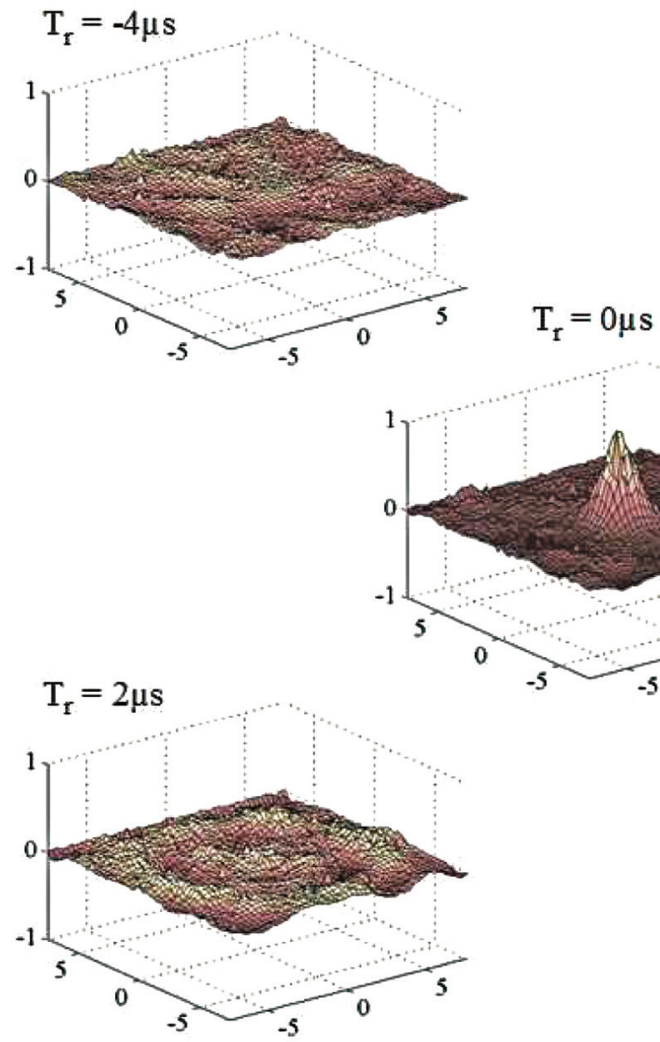

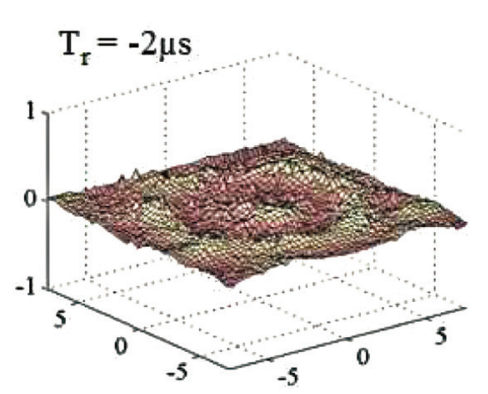

FIG. 6. (Color online) Inverse Filter (IF) recompression experiment on a rectangular steel plate equipped with a chaotic cavity transducer: Snapshots of the 2D scan near the intended focal point $(7.5 \mathrm{~mm}$ away from the border) for 5 time instances around the focal time. moment of the time recompression. To prove that focusing is only possible using a CCT, the experiment was repeated using a single PZT transducer (without intermediate chaotic cavity) as a source. As expected, the result simply is an outward propagating circular wave going from left to right, as can be seen in Fig. 8(a). Due to the geometry of the medium, the conducted waves are Lamb waves. Several modes, with different wave speeds, can clearly be seen in the figures. This is in agreement with experimentally measured "wavenumber-frequency" FFT results obtained on this plate, as discussed later in the paper.

The scans show that focusing can be obtained with the help of the CCT on nonreverberating samples, even though for obvious reasons the focusing quality is not as good as in the case of a reverberating sample. The contrast can be enhanced by showing the energy instead of the signal amplitude. The energy is defined as the sum of the squared signal


FIG. 7. Inverse Filter (IF) recompression experiment on a rectangular steel plate equipped with a chaotic cavity transducer: Spatial distributions of the focused signal in $x$ (dashed line) and $y$ (solid line) axis directions at the recompression time for a focal point near the border (a) and a focal point exactly on the border (b). The border is parallel to the $\mathrm{x}$ axis. amplitudes over a fixed time interval. In this case, the width of the temporal focus peak $(20 \mu \mathrm{s})$ was used as time interval. This method averages phase effects in the different time windows shown. As illustrated in Fig. 9, a distinctive focus spot becomes visible at full recompression time. The three above discussed figures also clearly reveal the strong effect of attenuation on the propagating waves.

When performing the above TR experiment using the chaotic cavity again, but now scanning two additional areas away from the focus zone [areas (b) and (c) in addition to zone (a) shown in Figs. 10 and 11], no traces of temporal or spatial focusing are seen. The snapshot of the energy at full recompression time (Fig. 10) illustrates that the energy is focused at the intended focal point inside area (a), whereas it is evenly spread over the other areas (b) and (c). As a means of verification, we repeated this experiment for a simple PZT transducer glued directly onto the plate. Obviously, the time reversal process then leads to circular waves equally propagating in all directions (Fig. 11).

Interestingly, in the case of nonreverberant media, the time evolution of the energy build-up at the focal point is completely different from the case of reverberant media. The focusing is achieved by propagating waves that solely come from the direction of the chaotic cavity transducer, and not from every direction around the focal spot, as is the case for a reverberant sample (see Fig. 6). For nonreverberant media, the sample does not contribute to the focusing process since the boundaries are too far away and attenuation is too high to allow a significant contribution from reflected waves. The chaotic cavity itself is entirely responsible, and thus indispensable, for the focusing. 


$$
\mathrm{t}=-10 \mu \mathrm{s}
$$
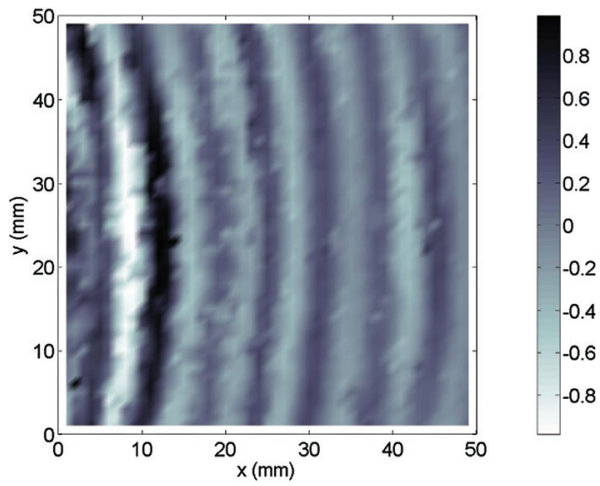

$$
t=0 \mu s
$$
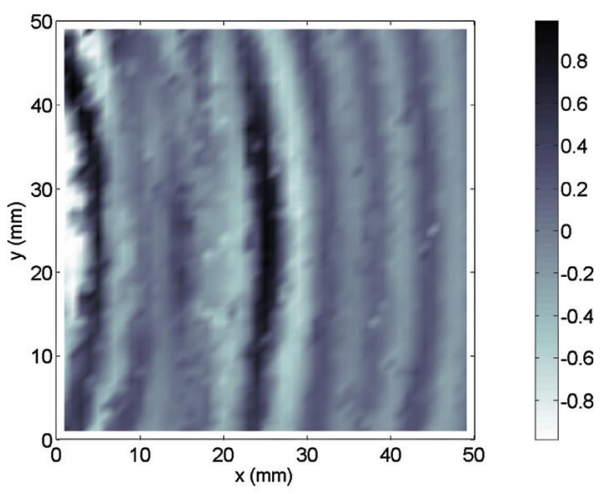

$$
\mathrm{t}=+10 \mu \mathrm{s}
$$

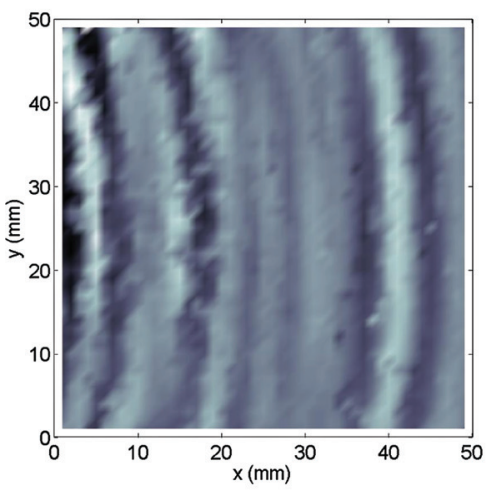

(a)

$$
t=-10 \mu s
$$

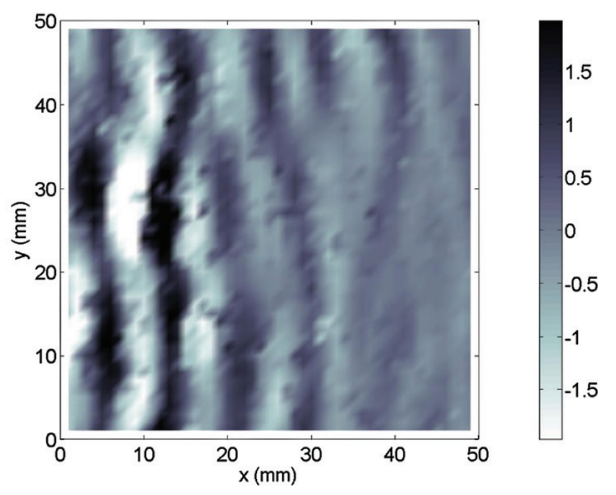

$$
t=0 \mu s
$$

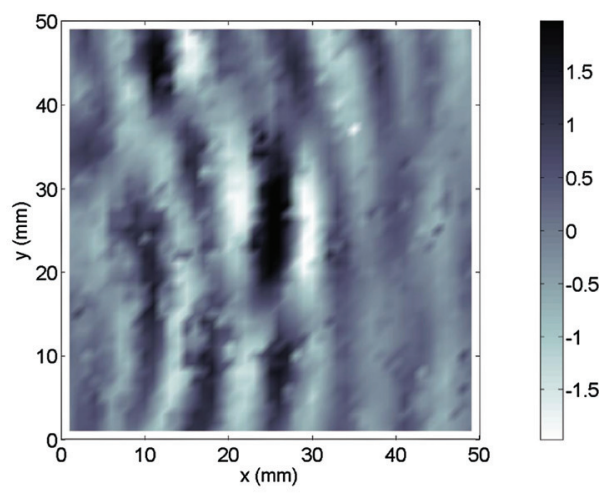

FIG. 8. (Color online) Inverse Filter (IF) recompression experiment on a large rectangular composite plate equipped with a a single PZT transducer (a) and a chaotic cavity transducer (b): 2D amplitude scans around an intended focal point at three instances in time: before, at, and after the focus time.

\section{$\mathrm{t}=+10 \mu \mathrm{s}$}

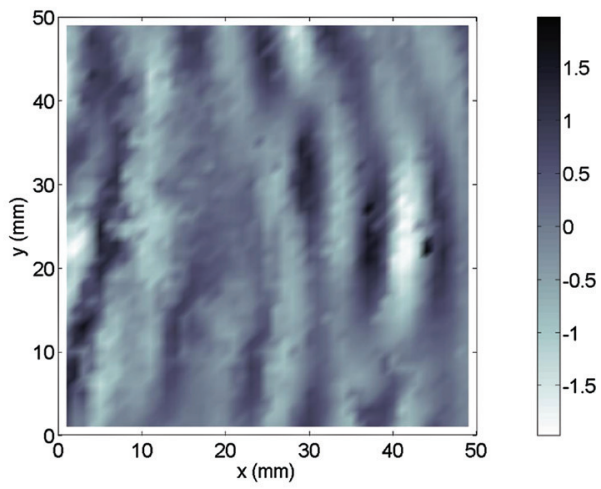

(b)

We substantiate this particular way of energy built-up at the focus by means of the following supplementary validation experiment in which we prove that a chaotic cavity transducer has similar properties as a multi-element transducer. It is known that multi-element focusing transducers provide a spatial resolution described by:

$$
\Delta R=\lambda \frac{F}{D}
$$

where $D$ is the width of the complete transducer in the direction perpendicular to the direction of the focus, $F$ the dis- tance between the transducer and the intended focal point and $\lambda$ is the wavelength of the dominant transmitted mode. This diffraction formula predicts that the focus width increases with the distance to the transducer. The spatial resolution formula (3) was tested for a CCT mounted on two composite plates: the large $\left(65 \times 65 \times 0.3 \mathrm{~cm}^{3}\right)$ composite plate used for the above discussed measurements, and a smaller $\left(35 \times 35 \times 0.2 \mathrm{~cm}^{3}\right)$ composite plate with less attenuation. The CCT consists of an irregular aluminum plate with holes, and a PZT disk. The CCT-plate contact area is $6 \mathrm{~cm} \times 1 \mathrm{~cm}$. A CCT-TR focusing experiment was performed for increasing distance from the CCT (on a line 
$\mathrm{t}=-10 \mu \mathrm{s}$

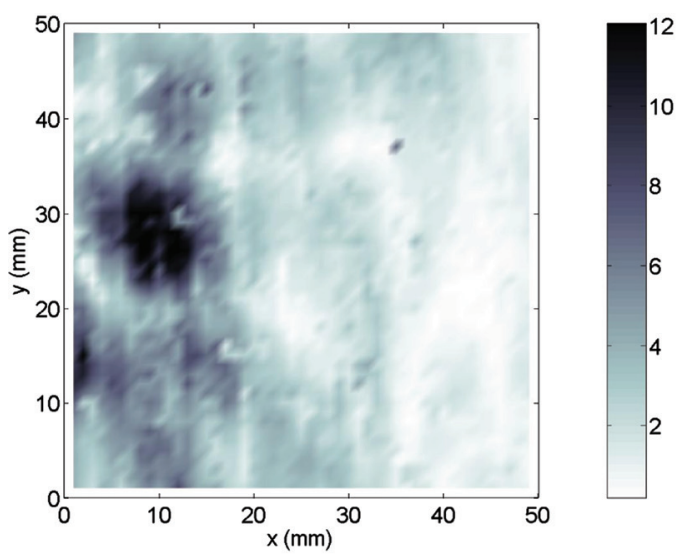

\section{$t=0 \mu s$}

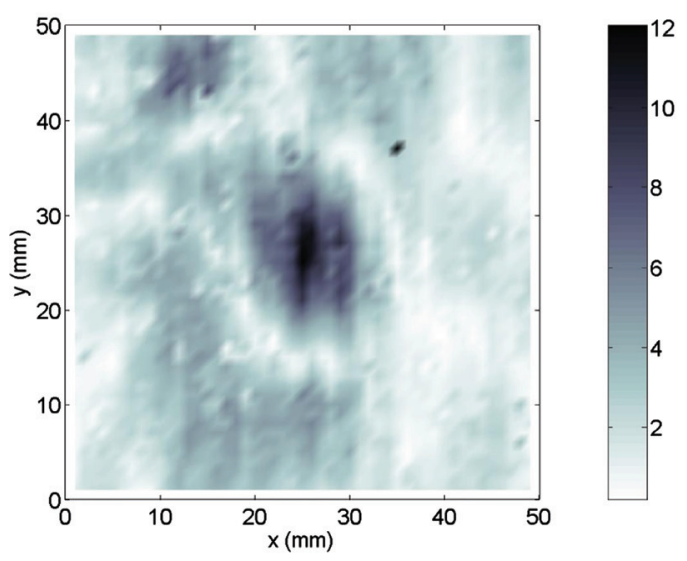

\section{$t=+10 \mu s$}

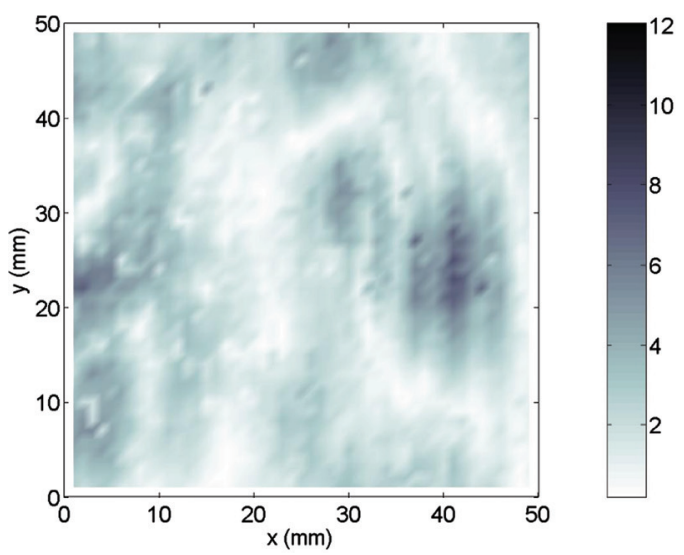

FIG. 9. (Color online) Inverse Filter (IF) recompression experiment on a large rectangular composite plate equipped with a chaotic cavity transducer: 2D energy scans around an intended focal point at three instances in time: before, at, and after the focus time.

perpendicular to the largest contact line of the CCT). At each distance, a linear scan parallel to the CCT orientation was performed, and the width of the energy focus was derived by extracting the half max-full width of a parabolic fit of the scan data.
This observed behavior for the larger plate is shown in Figs. 12(a) and 12(b). The width of the focus is indeed proportional to the distance from the transducer. In the smaller plate [Figs. 12(c) and 12(d)], the focus width remains more or less constant, whatever the distance to the chaotic cavity is. The latter result can be explained by the presence of, however, very small reflections in the smaller plate that contribute to the TR process, and build-up the energy focus with information coming from all directions. The existence of reflections can be evidenced by carefully analyzing the time movies made from the measured signals, or by quantifying them by means of a two dimensional Fourier transform analysis. ${ }^{28}$ The second method is frequently used to calculate dispersion curves in platelike structures, but can also distinguish the right and left running waves in the plate. To achieve this, a small PZT transducer, acting as a point source and placed in the center of the plate, emits short pulses (1-2 periods, windowed to avoid sidebands) of which the response is scanned by a laser vibrometer over a line perpendicular to one of the plate's borders. Using a small displacement step, sufficient information is provided to calculate the spatial, as well as the temporal, Fourier transform of the recorded signals in terms of wavenumber $k$ and frequency $f$. In this case, 1024 points with a $0.1 \mathrm{~mm}$ spacing along a straight line were scanned. The measured signal duration was 4096 points, for a sampling rate of $10 \mathrm{MHz}$. After a coordinate transformation of the Fourier transformed $k(f)$ results, a $v(f)$-relation can be obtained, both for positive and negative values of the wave speed $v$. Based on these results (not shown, but available from the authors), we could affirm that the left running (reflected) waves in the larger plate are almost inexistent, with an energy about $10^{4}$ times less than that of the right running waves. In the smaller plate, the energy of the reflected waves is only 1 or 2 orders of magnitude smaller than the direct waves. This means that even the smallest presence of reflections enhances the time reversal capabilities of the chaotic cavity but completely changes the energy build-up at the focus.

\section{Synthetic time reversal}

The new concept of a chaotic cavity transducer establishes an economical alternative for a phased array transducer. Phased arrays are multi-element transducers that can produce focused beams using electronic delaying of the source signals. ${ }^{29}$ They are widely used in biomedical imaging $^{30}$ and therapeutic treatment. ${ }^{31}$ Since these devices consist of several tens to hundreds of transducers that have to be triggered very precisely, they are rather expensive.

In order to use a chaotic cavity as a phased array transducer, the following steps are necessary. We start by acquiring the response signals in discrete recording points along a line, near and parallel to the cavity's largest dimension. Subsequently, the signals are convoluted with the time reversed emitted signal, time reversed again, and stored in a library on a computer. Due to the reciprocity and linearity of the system, simply adding these signals and sending them back through the cavity will result in a simultaneous energy focusing in all previously considered recording points. The energy 


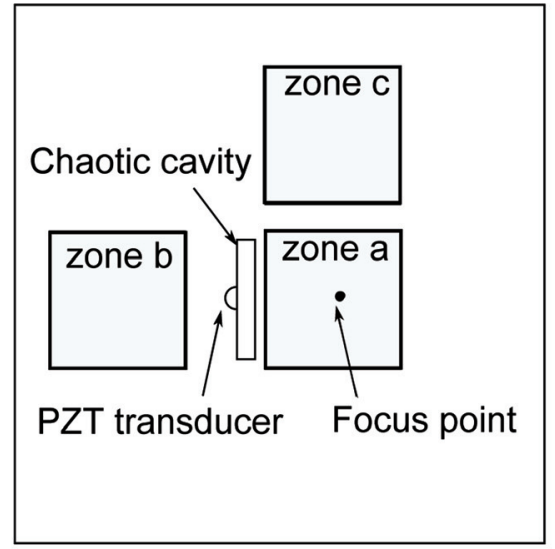

(c)
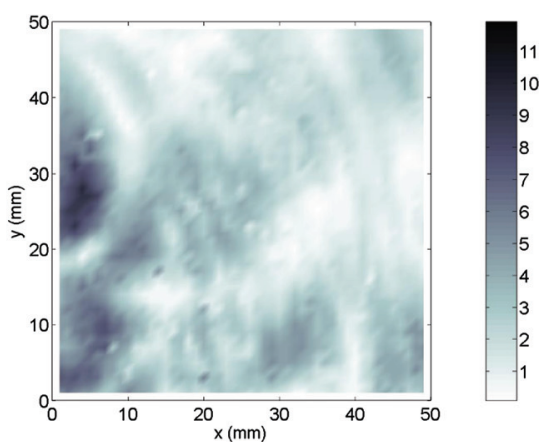

(b)

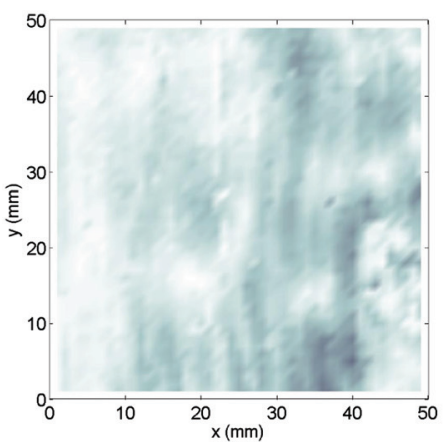

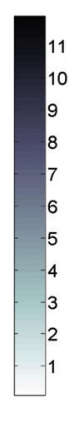

(a)

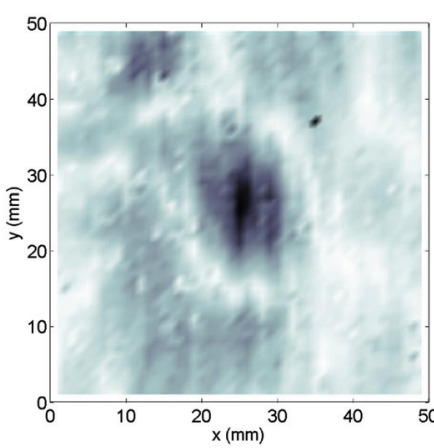

FIG. 10. (Color online) Chaotic cavity recompression experiment in a composite plate: energy at the focus time for three different zones a, b and c as indicated on the inset. at each focal point depends on the amount of signals considered in the rebroadcasted sum, since the total energy produced by the single transducer is normalized. After the moment of focusing, each point will act as a new secondary point source: a virtual source. If the focusing at all points is simultaneous, i.e., when all signals are summed and rebroad- casted without relative delays, this will create a plane wave in the direction perpendicular to the recording line. When relative delays are introduced before summing the signals, the time of focus in every virtual point source will be different. In order to focus energy at an arbitrary point on the plate, the delays have to be chosen such that the virtual point

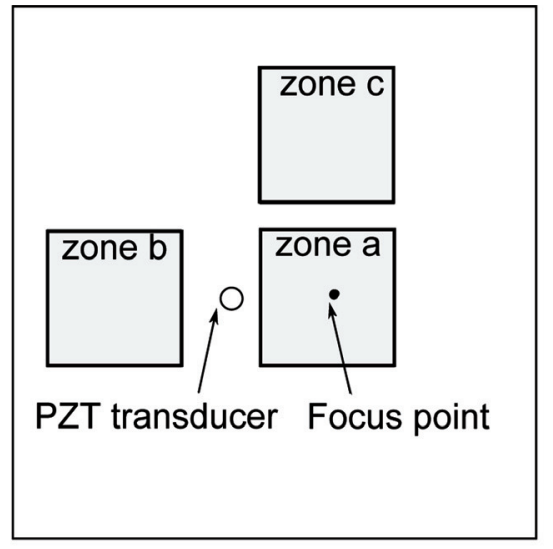

(b)

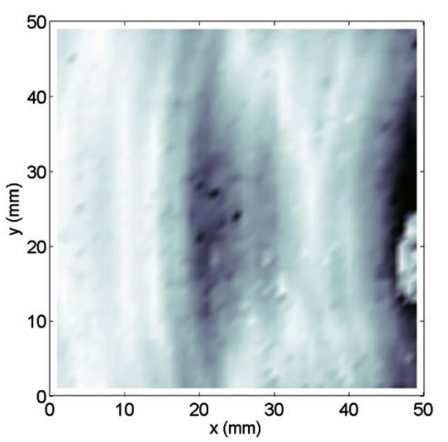

(c)


(a)

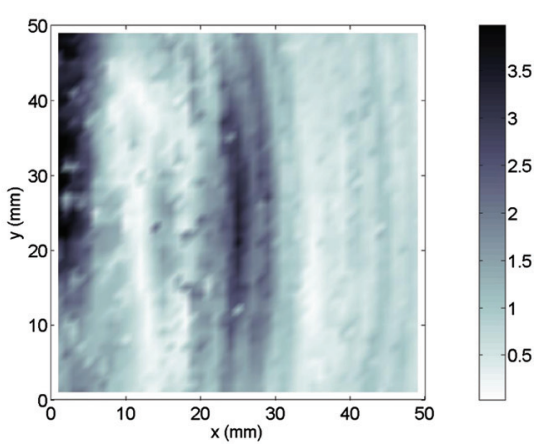

FIG. 11. (Color online) Single PZT transducer recompression experiment in a composite plate: energy at the focus time for three different zones $\mathrm{a}, \mathrm{b}$ and $\mathrm{c}$ as indicated on the inset. 
(a)

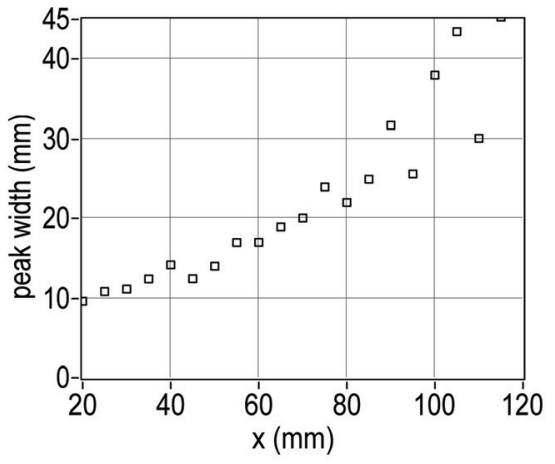

(c)

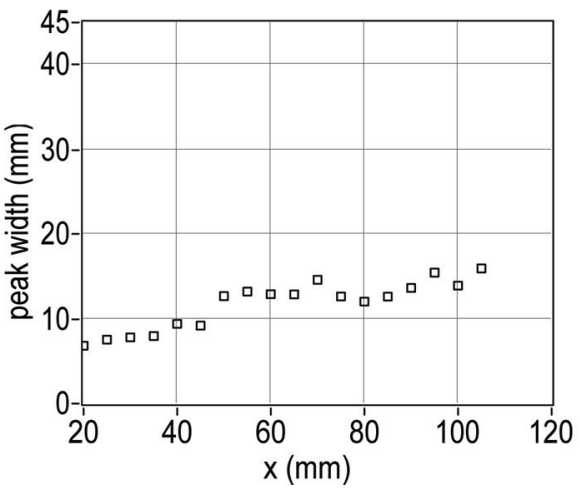

(b)

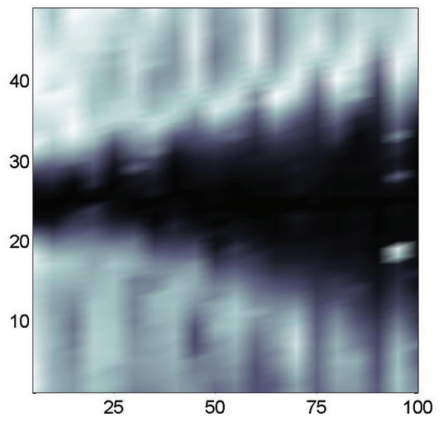

(d)

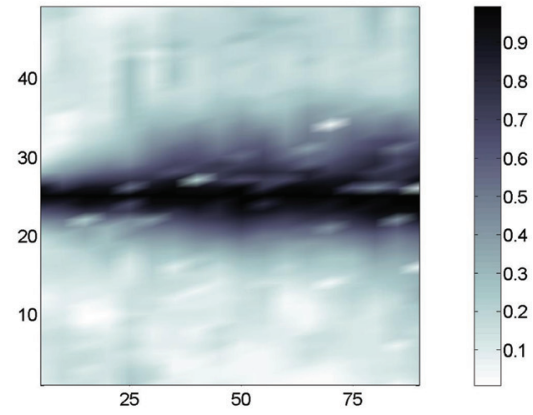

FIG. 12. (Color online) Comparison of the peak width as a function of the distance $x$ to the chaotic cavity in the large (a and b) and the small (c and d) plate. sources create circular wave fronts that arrive in the desired point exactly at the same focal time. Source points farther away from the intended focal point will have to be triggered earlier than source points nearby. To achieve this in experimental conditions, the library signals are first interpolated and shifted over the appropriate delay time which can be calculated as the ratio of the distance between the virtual source point and the intended focal point divided by the wave speed. Finally, after time shifting (which can be automated in LabView), the signals are added and re-emitted. Figure 13(a) shows an example of such a composed source signal, based on the received and delayed signals at 5 points close to the CCT contact line, and for an intended focal point $50 \mathrm{~mm}$ away from the cavity. The resulting temporal signal at the focal point, after rebroadcasting the composed signal from the CCT, is displayed in Fig. 13(b). The spatial distribution of the energy is visualized in Fig. 14.

The major advantage of this technique over a traditional phased array transducer is the low cost of the system, since only one transducer is necessary, and no demanding electronical synchronization is used.

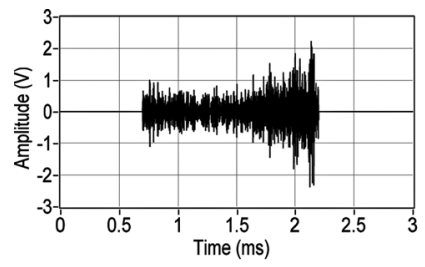

(a)

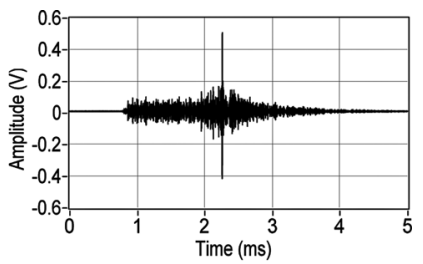

(b)
FIG. 13. Source signal (a) and time reversed signal (b) generated by a synthetic time reversal experiment in a polymer plate. A library of 5 points was used to focus energy $50 \mathrm{~mm}$ away from the cavity.
A joint disadvantage for both phased arrays and CCTsynthetic focusing is the fact that the wave speed distribution in the plate must be known exactly, in order to calculate the time shift of the different source signals. Especially in the case of composite plates this may lead to inaccuracies, since the wave speed distribution can be highly anisotropic. In our CCT synthetic focusing experiments, the contrast of the time reversed signal for focus points on various distances away from the cavity was optimized using different wave speeds ranging from 1200 to $2200 \mathrm{~m} / \mathrm{s}$. Moreover, for focal points away from the symmetry axis of the library source points, difficulties were experienced to achieve a good contrast, most probably related to the fact that different directions between virtual source points and focus point require different wave speeds. Indeed, when a constant wave speed value is used, the different waves will not arrive at the correct time

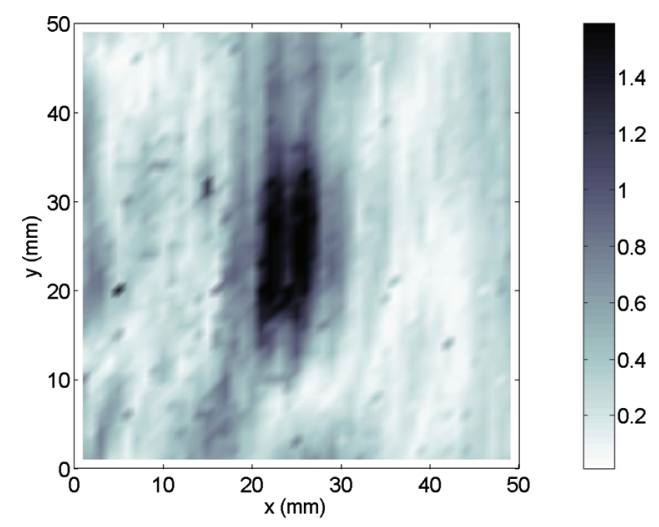

FIG. 14. (Color online) 2D scan of the focused energy of a chaotic cavity synthetic TR experiment in a polymer plate on the focus time. A library of 5 points was used to focus energy $50 \mathrm{~mm}$ away from the cavity. 
to interfere constructively with each other. Further research will be undertaken to clearly map out the anisotropy of the wave speeds in the plate, and to optimize the CCT synthetic focusing concept for practical uses.

\section{CONCLUSION}

In this paper, we have introduced the concept of "Chaotic Cavity Transducer" (CCT). The benefit of using a CCT is clearly to be found in the ability to focus energy at an arbitrary point of a solid structure. Unambiguous focal points can be obtained in media with high levels of symmetry as well as for nonreverberant media. In addition, two methods, Chirped excitation and Inverse Filtering, have been discussed to improve both the quality of the focusing and the contrast (signal to noise ratio). The spatial resolution properties of the CCT for focusing in nonreverberant media are found to be similar to multi-element transducers. Moreover, the CCT concept can be used to mimic a phased array transducer, with a possibility to focus energy in an arbitrary point of a medium, at a much lower cost. We believe the CCT concept also opens possibilities for high resolution imaging of nonlinear defects. ${ }^{32}$

${ }^{1}$ M. Fink, D. Cassereau, A. Derode, C. Prada, P. Roux, M. Tanter, J. Thomas, and F. Wu, Rep. Prog. Phys. 63, 1933 (2000).

${ }^{2}$ D. Cassereau and M. Fink, IEEE Trans. Ultrason. Ferroelectr. Freq. Control 39, 579 (1992).

${ }^{3}$ A. Derode, P. Roux, and M. Fink, Phys. Rev. Lett. 75, 4206 (1995).

${ }^{4}$ G. Montaldo, P. Roux, A. Derode, C. Negreira, and M. Fink, J. Acoust. Soc. Am. 110, 2849 (2001).

${ }^{5}$ P. Roux, B. Roman, and M. Fink, Appl. Phys. Lett. 70, 1811 (1997).

${ }^{6}$ P. Roux and M. Fink, J. Acoust. Soc. Am. 107, 2418 (2000).

${ }^{7}$ C. Draeger and M. Fink, Phys. Rev. Lett. 79, 407 (1997).

${ }^{8}$ C. Draeger, J. Aime, and M. Fink, J. Acoust. Soc. Am. 105, 618 (1999).
${ }^{9}$ C. Draeger and M. Fink, J. Acoust. Soc. Am. 105, 611 (1999).

${ }^{10}$ M. Fink and J. Rosny, Nonlinearity 15, R1 (2002).

${ }^{11} \mathrm{~N}$. Quieffin, "Etude du rayonnement acoustique de structures solides: vers un système d'imagerie haute résolution," Ph.D. thesis, Université Paris VI, 2004.

${ }^{12} \mathrm{~N}$. Quieffin, S. Catheline, R. Ing, and M. Fink, in Proceedings of the IEEE Ultrasonic Symposium, IEEE Montreal, (2004) pp. 801-804.

${ }^{13}$ K. Van Den Abeele, A. Sutin, J. Carmeliet, and P. Johnson, NDT E Int. 34, 239 (2001).

${ }^{14}$ R. Guyer and P. Johnson, Phys. Today 52(4), 30 (1999).

${ }^{15}$ I. Solodov, J. Wackerl, K. Pfleiderer, and G. Busse, Appl. Phys. Lett. 84, 5386 (2004).

${ }^{16}$ I. Solodov and G. Busse, Appl. Phys. Lett. 91, 251910 (2007).

${ }^{17}$ B. Sarens, G. Kalogiannakis, C. Glorieux, and D. Van Hemelrijck, Appl. Phys. Lett. 91, 264102 (2007).

${ }^{18}$ A. Sutin, J. TenCate, and P. Johnson, J. Acoust. Soc. Am. 116, 2779 (2004).

${ }^{19}$ T. Ulrich, P. Johnson, and R. Guyer, Phys. Rev. Lett. 98, 104301 (2007).

${ }^{20}$ T. Goursolle, S. Dos Santos, O. Bou Matar, and S. Calle, Int. J. Non-Linear Mech. 43, 170 (2008).

${ }^{21}$ T. Goursolle, S. Callé, S. Dos Santos, and O. Bou Matar, J. Acoust. Soc. Am. 122, 3220 (2007).

${ }^{22}$ G. Montaldo, D. Palacio, M. Tanter, and M. Fink, Appl. Phys. Lett. 84, 3879 (2004).

${ }^{23} \mathrm{~T}$. Misaridis and J. Jensen, IEEE Trans. Ultrason. Ferroelectr. Freq. Control 52, 177 (2005).

${ }^{24}$ K. Van Den Abeele, P. Le Bas, B. Van Damme, and T. Katkowski, J. Acoust. Soc. Am. 126, 963 (2009).

${ }^{25}$ M. Tanter, J. Aubry, J. Gerber, J. Thomas, and M. Fink, J. Acoust. Soc. Am. 110, 37 (2001).

${ }^{26}$ J. Aubry, M. Tanter, J. Gerber, J. Thomas, and M. Fink, J. Acoust. Soc. Am. 110, 48 (2001)

${ }^{27}$ A. Derode, A. Tourin, and M. Fink, J. Appl. Phys. 85, 6343 (1999).

${ }^{28}$ W. Prosser, M. Seale, and B. Smith, J. Acoust. Soc. Am. 105, 2669 (1999).

${ }^{29}$ O. Von Ramm and S. Smith, IEEE Trans. Biomed. Eng. 438 (2007).

${ }^{30}$ J. Kisslo, O. VonRamm, and F. Thurstone, Circulation 53, 262 (1976).

${ }^{31}$ K. Hynynen, G. Clement, N. McDannold, N. Vykhodtseva, R. King, P. White, S. Vitek, and F. Jolesz, Magn. Reson. Med. 52, 100 (2004).

${ }^{32}$ O. Bou Matar, Y. F. Li, K. Van Den Abeele, Appl. Phys. Lett. 95, 141913 (2009). 